\title{
Effects of iota-carrageenan on ocular Chlamydia trachomatis infection in vitro and in vivo
}

\author{
Aleksandra Inic-Kanada ${ }^{1}$ - Elisabeth Stein ${ }^{1}$ - Marijana Stojanovic ${ }^{2} \cdot$ Nadine Schuerer $^{1}$ • Ehsan Ghasemian ${ }^{1}$. \\ Ana Filipovic $^{2} \cdot$ Emilija Marinkovic $^{2} \cdot$ Dejana Kosanovic $^{2} \cdot$ Talin Barisani-Asenbauer $^{1}$ (D)
}

Received: 21 June 2017 /Revised: 16 February 2018 / Accepted: 27 February 2018 /Published online: 13 March 2018

(C) The Author(s) 2018

\begin{abstract}
Ocular chlamydial infections with the ocular serovars A, B, Ba, and C of Chlamydia trachomatis represent the world's leading cause of infectious blindness. Carrageenans are naturally occurring, sulfated polysaccharides generally considered safe for food and topical applications. Carrageenans can inhibit infection caused by a variety of viruses and bacteria. To investigate whether iota-carrageenan (I-C) isolated from the red alga Chondrus crispus could prevent ocular chlamydial infection, we assessed if targeted treatment of the conjunctival mucosa with I-C affects chlamydial attachment, entry, and replication in the host cell. Immortalized human conjunctival epithelial cells were treated with I-C prior to C. trachomatis infection and analyzed by flow cytometry and immunofluorescence microscopy. In vivo effects were evaluated in an ocular guinea pig inclusion conjunctivitis model. Ocular pathology was graded daily, and chlamydial clearance was investigated. Our study showed that I-C reduces the infectivity of $C$. trachomatis in vitro. In vivo results showed a slight reduced ocular pathology and significantly less shedding of infectious elementary bodies by infected animals. Our results indicate that I-C could be a promising agent to reduce the transmission of ocular chlamydial infection and opens perspectives to develop prophylactic approaches to block C. trachomatis entry into the host cell.
\end{abstract}

Keywords Carrageenan $\cdot$ Trachoma $\cdot$ Natural products $\cdot$ Chlamydia

\section{Introduction}

Ocular (Lietman et al. 1998) and genital (Norman 2002) chlamydial infections are common worldwide (146 million cases/ year) (ECDC 2015). Repeated infections with Chlamydia trachomatis ocular serovars A, B, Ba, and C trigger fibrotic processes in the affected ocular tissues leading to trichiasis, corneal opacity, and in further progression to complete loss of sight (Mariotti et al. 2009). Apart from reducing the quality of life, ocular chlamydial infections can lead to a sight-threatening complication called trachoma. Trachoma is considered a neglected

Talin Barisani-Asenbauer

talin.barisani@meduniwien.ac.at

1 OCUVAC - Center of Ocular Inflammation and Infection, Laura Bassi Centres of Expertise, Center for Pathophysiology, Infectiology and Immunology, Medical University of Vienna, Kinderspitalgasse 15, 1090 Vienna, Austria

2 Department of Research and Development, Institute of Virology, Vaccines and Sera - TORLAK, Belgrade, Serbia tropical disease and is the world's leading infectious cause of preventable blindness (Taylor et al. 2014). Currently, control in the endemic populations is achieved through mass drug administration (Frick et al. 2001) and implementation of the SAFE strategy ( $S$ stands for "surgery" for trachomatous trichiasis, $A$ stands for "antibiotic" treatment, $F$ stands for "facial" cleanliness, and $E$ stands for "environmental" improvement such as general community hygiene, adequate water supply, and construction of sanitary facilities) (WHO 2014).

To more efficiently combat the spread of $C t$ in these rural poverty areas, novel methods for treatment and prevention of C. trachomatis infection are needed. There exist various studies exploring the potential of natural products for developing new anti-chlamydial treatment modalities (Brown et al. 2016). Polyphenolic (Daglia 2012), lipidic (Bergsson et al. 1998), proteinaceous compounds (Yasin et al. 1996), and polyherbal formulations have demonstrated significant anti-chlamydial activity (Talwar et al. 1995). Overall, natural products show significant potential in treating chlamydial infections and the development of novel drugs based on natural products may help in the global management of Chlamydiae-related infections. 
Carrageenans are naturally occurring anionic sulfated polysaccharides (SPs), which are present in a number of seaweeds of the class Rhodophyceae, such as Chondrus, Gigartina, Hypnea, and Eucheuma (Lahaye 2001; Necas and Bartosikova 2013). They have an excellent and well-documented safety profile for long-term use, since they have been widely used in the food, pharmaceutical, and cosmetic industry as additives, thickeners, and emulsifiers. The Food and Drug Administration has listed carrageenan compounds as "generally recognized as safe" (GRAS) in 1973 (FDA SCOGS (Select Committee on GRAS Substances) n.d.). Carrageenans consist of alternate units of Dgalactose and 3,6-anhydro-galactose, both sulfated and nonsulfated, joined by $\alpha-1,3$ and $\beta-1,4$-glycosidic linkage (Necas and Bartosikova 2013). Depending on the allocation of the sulfate groups on the main structures, carrageenan is classified into various types $(\lambda, \kappa, \iota, \varepsilon, \mu)$, all containing 22 to $35 \%$ sulfate groups, of which $\lambda, \kappa$-, and $\iota$-carrageenans are widely used in the food industry (Vera et al. 2011; Necas and Bartosikova 2013).

Different types of carrageenans have been found to be active against a variety of viruses, including human papillomavirus (HPV) (Buck et al. 2006; Roberts et al. 2007; Levendosky et al. 2015), herpes simplex virus (Carlucci et al. 1999), rhinovirus (Grassauer et al. 2008), influenza (Leibbrandt et al. 2010), metapneumovirus (Klimyte et al. 2016), and rabies (Luo et al. 2015). Antiviral potential against human immunodeficiency virus was already described two decades ago (Gonzalez et al. 1987) and has been reviewed recently (Damonte et al. 2004). Although it has been proven that carrageenans show strong antiviral properties, the exact mechanisms of action and structural determinants for these compounds are not fully elucidated. It has been hypothesized that carrageenans exert their antiviral activity by direct interaction with virus particles at an early stage of viral infection (Gonzalez et al. 1987). Initial attachment of the virus to human cells is mediated by the interactions between the virion and a type of cell surface glycosaminoglycan (GAG) heparan sulfate (Buck et al. 2006). Carrageenans closely resemble heparan sulfate and could interact directly with the viral particles, preventing attachment to the respective receptors on the cell surface. It has also been shown that iota-carrageenan (I-C) possesses antiviral activity not only due to direct interaction with influenza A virus but also due to coating of cellular structures, hindering receptor binding sites (Buck et al. 2006; Leibbrandt et al. 2010).

The effects of carrageenans also have been described in the context of bacterial infections. The antimicrobial action of I-C on food-borne pathogenic bacteria has been described and shown that the inhibitory effect of carrageenans was not bactericidal but bacteriostatic (Yamashita 2001). Moreover, the removal of sulfate residues eliminated the bacteriostatic effect of I-C, suggesting that the sulfate residue(s) in carrageenan play an essential role in this mechanism.
Studies investigating the effects of SPs to block chlamydial infection are sparse. It has been shown that exogenous heparin and heparan sulfate inhibit chlamydial infection (Zhang and Stephens 1992). Although this study used C. trachomatis lymphogranuloma venereum serovar L2 as their model organism, their results might be translatable to ocular strains since it was shown that for both serovariants heparan sulfate-like-mediated interactions between $C$. trachomatis and eukaryotic cells are critical mediators of infectivity (Chen and Stephens 1994). Moreover, the ability of different SPs to inhibit the infection of cervix derived human epithelial cells ME180 with C. trachomatis serovar $E$ was studied and included different types of carrageenan and GAGs (e.g., heparin, heparan sulfate, and hyaluronic acid). This in vitro study was important as it demonstrated that I-C and other types of SPs block infection of ME180 with $C$. trachomatis serovar E by preventing bacterial attachment to the host cell (Zaretzky et al. 1995). This is in line with numerous reports showing that the entry of Chlamydia into the host cell is dependent on elementary bodies (EBs) interactions with GAGs exposed on the surface of the host cells (Chen and Stephens 1994; Moelleken and Hegemann 2008).

It is well known that a successful prevention of an infection depends on how efficient a certain product/ drug is in preventing the entry of the infectious agent into the host organism. For chlamydial infections, this would mean inhibiting/preventing the infection of ocular and genital mucosal epithelium. Most importantly, even before taking any product/drug into consideration for treatment of chlamydial infections, their safety profiles on mucosal surfaces must be established. Recently, it has been shown that carrageenans, including I-C, were not cytotoxic and did not induce proinflammatory cytokines in epithelial cell lines HT-29 and HCT-8 (McKim Jr. et al. 2016). Moreover, in studies investigating possibilities of using carrageenans as microbicides, it has been shown that a topical application of carrageenan is safe for mucosal epithelium (Coggins et al. 2000; Grassauer et al. 2008; Eccles et al. 2015).

In the present study we used (i) an in vitro infection model highly resembling the human ocular surface: immortalized human conjunctival epithelial (HCjE) cells infected with $C$. trachomatis ocular serovar B (CtB) (Stein et al. 2013; Rahn et al. 2016) and (ii) an in vivo guinea pig inclusion conjunctivitis infection model, which uses Chlamydia caviae (GPIC), a natural pathogen of the guinea pigs, as the infectious agent (Rank and Whittum-Hudson 1994; Belij-Rammerstorfer et al. 2016; Filipovic et al. 2017) to investigate if targeted treatment of the ocular conjunctival mucosa with I-C derived from Chondrus crispus can block chlamydial attachment, entry, and by these means also bacterial replication in the ocular epithelial cells. 


\section{Material and methods}

\section{Chlamydial strains}

Chlamydia trachomatis ocular serovar B (CtB) (ATCC VR573) and Chlamydia caviae (GPIC) (kindly provided by Prof. Roger G. Rank) were propagated in McCoy cells (ATCC CRL-1696) according to standard procedures (Caldwell et al. 1981). Harvested stocks were centrifuged at $200 \times g$ to pellet cellular debris, resuspended in sucrose-phosphateglutamate buffer (SPG) containing $0.01 \mathrm{M}$ sodium phosphate (pH 7.2), $0.25 \mathrm{M}$ sucrose, and $5 \mathrm{mM} \mathrm{L-glutamic}$ acid.

\section{Cell culture}

HCjE cells, kindly provided by Prof. Ilene Gipson (Schepens Eye Research Institute, Harvard Medical School, Boston), were maintained in keratinocyte serum-free medium supplemented with bovine pituitary extract, $0.2 \mathrm{ng} \mathrm{mL}^{-1}$ EGF and $1 \%$ penicillin/streptomycin (Life Technologies, UK) at $37^{\circ} \mathrm{C} /$ $5 \% \mathrm{CO}_{2}$ and $95 \%$ humidity. The medium was changed every second day, and cells were passaged at $70 \%$ confluence.

\section{In vitro infection assay}

For in vitro infection assays, $\mathrm{HCjE}$ cells were maintained in keratinocyte serum-free medium (Life Technologies, UK) at $37^{\circ} \mathrm{C} / 5 \% \mathrm{CO}_{2}$ and $95 \%$ humidity. The medium was changed every second day, and the cells were passaged at $70 \%$ confluence. Cells were harvested by trypsinization $(0.05 \%$ Trypsin/ $0.02 \%$ EDTA in PBS, GE Healthcare) and seeded at a density of 150.000 cells per well in 24-well plates (Greiner Bio-One, Austria). An aqueous solution containing $2.4 \mathrm{mg} \mathrm{mL}^{-1} \mathrm{I}-\mathrm{C}$ (0.24\% w/v; I-C derived from Chondrus crispus; FMC Biopolymers, USA) and $36.4 \mathrm{mg} \mathrm{mL}^{-1}$ mannitol (Sigma Aldrich, Germany) was formulated to test the antimicrobial activity against $\mathrm{CtB}$. The dose was chosen according to the results of a preliminary experiment $-2.4 \mathrm{mg} \mathrm{mL}^{-1}$, which was the concentration of an obtained liquid solution, was the most efficient dose in inhibiting chlamydial infection (serial dilutions $2.4,1.2,0.6$, and $0.3 \mathrm{mg} \mathrm{mL}^{-1}$ ) showed lower dosedependent inhibiting effect and all doses did not exhibit any cytotoxicity on HCjE cells. The I-C solution was filtered through a $0.22-\mu \mathrm{M}$ (Sarstedt, Germany) sterile filter and stored at $4{ }^{\circ} \mathrm{C}$. The unbuffered I-C solution had a $\mathrm{pH}$ between 6.8 and 7.4 and an osmolarity between 210 and $220 \mathrm{mOsm} \mathrm{kg}{ }^{-1}$. A sterile aqueous solution containing $36.4 \mathrm{mg} \mathrm{mL}^{-1}$ mannitol served as a placebo control.

Confluent cultures of $\mathrm{HCjE}$ cells were treated with I-C solution in the given concentration and subsequently infected with $1 \times 10^{4}$ inclusion-forming unit (IFU) $\mathrm{CtB}$, resuspended in SPG, per well. Placebo-treated cells and cells without any pre-treatment served as controls. A second group of mock- infected cells, treated only with either I-C or placebo, were assessed in the same manner to evaluate possible cytopathic effects of the formulations by phase-contrast microscopy. $\mathrm{HCjE}$ cells were incubated for $2 \mathrm{~h}$ at $37{ }^{\circ} \mathrm{C}$ to ensure $\mathrm{CtB}$ attachment. After $2 \mathrm{~h}$, the medium was changed to standard growth medium without antibiotics and cells were incubated for $48 \mathrm{~h}$ at $37^{\circ} \mathrm{C} / 5 \% \mathrm{CO}_{2}$ and $95 \%$ humidity. Cells were fixed with ice-cold methanol for $20 \mathrm{~min}$ at $-20^{\circ} \mathrm{C}$, air-dried, and stained with an anti-Chlamydia LPS-FITC labeled antibody (Clone B410F, Pierce Biotechnology, USA) diluted 1:20 in PBS for 30 min at $37^{\circ} \mathrm{C}$. CtB IFUs were counted on a Zeiss AxioObserver microscope using Tissue FaxSi Software (Tissuegnostics, Austria) for acquisition. $\mathrm{HCjE}$ cells were counterstained with DAPI. Inclusion size was measured in 20 high power fields per sample. All experiments were repeated three times and were performed in triplicates.

\section{Adhesion assay}

To assess changes in the efficiency of attachment of $\mathrm{CtB}$ elementary bodies (EBs) to the cell surface of I-C-treated $\mathrm{HCjE}$ cells, a flow cytometric adhesion assay with carboxyfluorescein succinimidyl ester (CFSE) labeled CtB EBs was performed according to a previous study (Molleken et al. 2010). Briefly, CtB EBs resuspended in SPG were labeled with $20 \mu \mathrm{mol} \mathrm{L}{ }^{-1}$ CSFE (ebioscience, Vienna Austria) for $90 \mathrm{~min}$ at room temperature (RT) as previously described (Schnitger et al. 2007). EBs were washed three times with PBS containing $1 \%$ BSA to remove excess CSFE. Confluent monolayers of $\mathrm{HCjE}$ cells (150.000 cells per well) were treated with $2.4 \mathrm{mg} \mathrm{mL}{ }^{-1} \mathrm{I}-\mathrm{C}$ or placebo solution for $5 \mathrm{~min}$ at $37{ }^{\circ} \mathrm{C} / 5 \%$ $\mathrm{CO}_{2}$ and $95 \%$ humidity. After $5 \mathrm{~min}$ of incubation of $\mathrm{HCjE}$ cells with I-C, labeled CtB EBs at a MOI (multiplicity of infection) of 10 were added into wells and further incubated for $1 \mathrm{~h}$ at $37{ }^{\circ} \mathrm{C} / 5 \% \mathrm{CO}_{2}$ and $95 \%$ humidity. Cells were then washed with PBS, harvested by trypsinization, and fixed with 2\% paraformaldehyde in PBS for 15 min at RT. Fluorescence of cells infected with CFSE-labeled CtB was assessed with a FACS Calibur flow cytometer (BD Biosciences, Germany). The experiment was repeated twice and both times was performed in triplicates.

\section{In vivo infection}

All animal experiments were approved by the Torlak Institute and conformed to the Serbian laws and European regulations on animal welfare (Approval No. 323-07-01577/2016-05/12). All animals were handled in strict accordance with good animal practice as defined by the Serbian code of practice (published in Sluzbeni Glasnik No. 41/9) for the care and use of animals for scientific purposes, the Guide for the Care and Use of Laboratory Animals of the Torlak Institute (2133/1, 21. 04. 2011), a Basel declaration that is committed to the $3 R$ 
principle (replace, reduce, refine). Every effort was made to minimize animal suffering. Any animals found to be requiring treatment were given appropriate veterinary care. We did not observe any unexpected deaths of animals during this study.

In vivo efficacy of I-C treatment was evaluated on guinea pigs Hartley Strain (300-350 g) anesthetized with a mixture of ketamine $\left(30 \mathrm{mg} \mathrm{kg}^{-1}\right)$ and xylazine $\left(2 \mathrm{mg} \mathrm{kg}^{-1}\right)$ applied intramuscularly prior any manipulation. Two sets of in vivo experiments were done. In the first experiment, we evaluated the impact of local I-C application prior GPIC inoculation on the course of chlamydial infection (pre-treatment). Guinea pigs were treated either with $25 \mu \mathrm{L}$ per eye of I-C $\left(2.4 \mathrm{mg} \mathrm{mL}^{-1}\right)$ or with appropriate placebo solution $2 \mathrm{~h}$ before the infection with $1 \times 10^{4}$ IFU of GPIC. In the second experiment, we evaluated the impact of local I-C application during the acute phase of infection (treatment). Guinea pigs infected with $1 \times$ $10^{4}$ IFU of GPIC were treated for 7 days, daily, either with $25 \mu \mathrm{L}$ per eye of I-C $\left(2.4 \mathrm{mg} \mathrm{mL}^{-1}\right)$ or with appropriate placebo solution starting from day 3 post-infection. The day of GPIC inoculation was considered as day 0 in both experiments. An experienced ophthalmologist who was blinded to the experimental groups observed and scored daily the eyes of guinea pigs in a protection assay (Rank et al. 1995). In brief, the palpebral and the bulbar conjunctivae were evaluated for erythema, edema, and exudation in each animal. Each observation was classified into five categories: (0.5) trace pathologic response, (1) slight erythema or edema of either the palpebral or the bulbar conjunctiva, (2) definite erythema or edema of either the palpebral or the bulbar conjunctiva, (3) definite erythema or edema of both the palpebral and the bulbar conjunctivas, or (4) definite erythema or edema of both the palpebral and the bulbar conjunctivas plus the presence of exudate.

\section{Determination of GPIC IFUs from conjunctival swabs}

For the quantification of GPIC EBs, conjunctival swab samples were collected from guinea pigs while under ketamine/ xylazine anesthesia before and $4,7,14$, and 21 days postinfection. Darcon swabs were used to swab the palpebral and the bulbar conjunctiva and placed in Copan Universal Transport Medium (UTM-RT) System (Copan, Italy). IFUs were determined by inoculation of the obtained swab material onto confluent cultures of McCoy cells (ATCC CRL-1696). Centrifugation at $200 \times g$ for $1 \mathrm{~h}$ was carried out to ensure attachment of EBs. After incubation for $24 \mathrm{~h}$ at $37^{\circ} \mathrm{C} / 5 \% \mathrm{CO}_{2}$ and $95 \%$ humidity in the presence of $1 \mathrm{mg} \mathrm{mL} \mathrm{L}^{-1}$ cyclohexamide (Sigma Aldrich) cells were fixed in ice-cold methanol and stained with a FITC-conjugated monoclonal antibody against Chlamydia LPS (1:20 in PBS, Clone B410F, Pierce Biotechnology, USA). IFUs were counted using a epifluorescence microscope (Zeiss AxioObserver, Zeiss, Germany).

\section{Statistical analysis}

To assess statistically significant differences between the treatment groups, a two-way ANOVA followed by Bonferroni multiple comparisons test was used. The level of significance was set at $P<0.05$. All statistical analyses were performed by the software: IBM SPSS Statistics 20.

\section{Results}

\section{I-C reduced the number of detectable inclusions in vitro}

The analyses of $\mathrm{HCjE}$ cells infected in vitro with $\mathrm{CtB}$ without any pre-treatment or upon I-C and placebo treatment encompassed the evaluation of $\mathrm{CtB}$ attachment, the counting of CtB IFUs, and the evaluation of inclusions' size.

Flow cytometric analyses of $\mathrm{HCjE}$ cells infected with CFSE-labeled CtB EBs revealed that I-C inhibits CtB attachment to the HCjE cells (Fig. 1). HCjE cells infected with CFSE-labeled $\mathrm{CtB}$, either without pre-treatment or upon placebo treatment were $\mathrm{CtB}$ positive $(\mathrm{CtB}+)(99.4 \pm 0.1 \%$ and $99.6 \pm 0.1 \%$ ) (Fig. 2a, b). Analysis of viable HCjE cells exposed to CFSE-labeled CtB in the presence of I-C revealed that I-C significantly impaired $\mathrm{CtB}$ attachment to $\mathrm{HCjE}$ cells because $65.1 \pm 2.8 \%$ of cells were $\mathrm{CtB}+$ (Fig. $2 \mathrm{a}$; $P<0.001$ compared either to $\mathrm{HCjE}$ cells infected with CFSE-labeled $\mathrm{CtB}$ without any pre-treatment or upon placebo treatment). Besides, I-C treatment resulted in a significant reduction of the CFSE signal: the mean fluorescence intensity (MFI) of $\mathrm{CtB}+\mathrm{HCjE}$ cells pre-treated with $\mathrm{I}-\mathrm{C}$ was $59.23 \pm 1.34$. MFI of $\mathrm{HCjE}$ cells pre-treated with I-C was significantly lower in comparison with MFIs recorded for HCjE cells treated either with placebo $(351.67 \pm 2.85, P<0.001)$ or without any treatment $(355.33 \pm 9.17, P<0.001)$ (Fig. $2 \mathrm{c}, \mathrm{d})$.

The counting of CtB IFUs in HCjE cells exposed to specific treatments prior in vitro $\mathrm{CtB}$ infection revealed significantly $(P<0.001)$ lower numbers of CtB IFUs for I-C-treated $\mathrm{HCjE}$ cells $\left(326 \pm 11 \mathrm{IFU}\right.$ well $\left.{ }^{-1}\right)$ in comparison with $\mathrm{CtB}-$ infected placebo-treated $\mathrm{HCjE}$ cells $(2403 \pm 89 \mathrm{IFU} /$ well $)$ (Fig. 3a, b). Moreover, inclusions observed upon in vitro $\mathrm{CtB}$ infection in I-C-treated HCjE cells $(5.35 \pm 0.38 \mu \mathrm{m})$ were significantly smaller $(P<0.001)$ in comparison to those in placebo-treated HCjE $(15.72 \pm 0.63 \mu \mathrm{m}$; Fig. 3a, c).

\section{The I-C-treated guinea pigs showed less severe symptoms of ocular chlamydial infection in vivo}

Preliminary performed experiments showed that topical treatment with I-C in guinea pigs was well tolerated, as any signs of irritation on the ocular surface were not marked during daily follow-ups. 


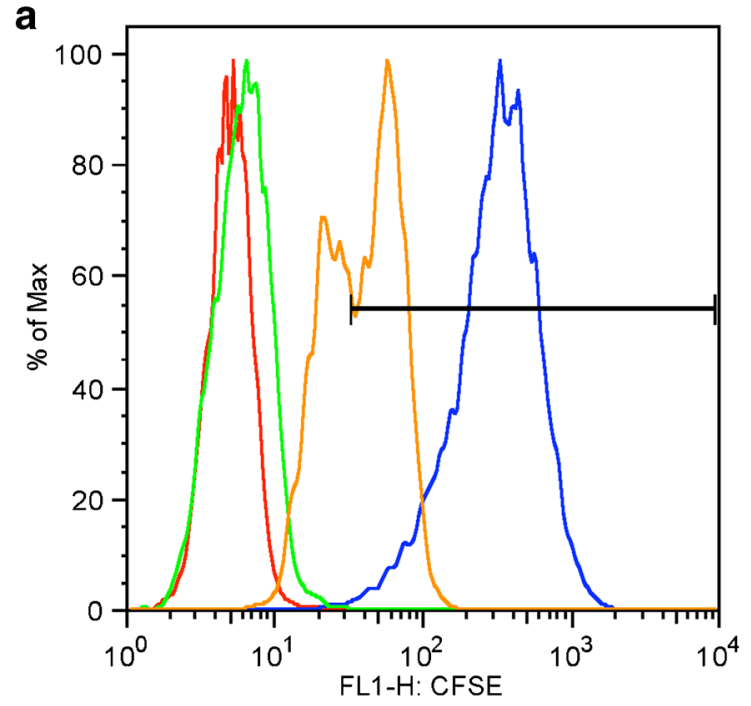

Fig. 1 Flow cytometric analyses of $\mathrm{HCjE}$ cells infected in vitro with CFSE-labeled CtB in the presence of I-C (a) or placebo (b) solution. Three independent samples per treatment were analyzed and representative histograms are presented. Each sample consisted of $1 \times 10^{6} \mathrm{HCjE}$ cells exposed to $\mathrm{CtB}$ at $\mathrm{MOI}$ of 10. CtB-infected $\mathrm{HCjE}$, which were not exposed to either I-C or placebo (blue line), non-infected $\mathrm{HCjE}$ cells (red

In comparison with placebo treatment, a single local I-C administration before GPIC ocular infection did not significantly alter the infection course. During the post-infection period, pathology scores were slightly lower in the I-C pretreated group compared to the animals that received placebo treatment, although statistically significant differences in intensity of pathology were not marked at any time-point (Fig. 4a). Furthermore, the peak of infection was reached on day 4 postinfection in both groups. The I-C-treated group showed a slightly delayed onset of pathology and less severe symptoms of infection up to day 16. By reaching day 21, all I-C-treated guinea pigs showed no more signs of any pathological events, whereas traces of inflammation were seen within the placebotreated group. Analysis of changes in chlamydial load during the post-infection period (Fig. $4 \mathrm{~b}$ ) revealed significantly lower absolute numbers of GPIC EBs in I-C-treated guinea pigs on day $4(P<0.001)$ and on day 7 post-infection $(P<0.001)$ compared to the corresponding placebo-treated animals. On day 14 post-infection, the number of GPIC EBs was also lower, but not statistically significant, in I-C-treated guinea pigs $(P>0.05)$. No GPIC IFUs could be detected in both groups by day 21 indicating complete clearance of chlamydial infection.

Multiple local I-C applications during the acute phase of ocular GPIC infection exerted more pronounced impact on infection intensity and pathologic response (Fig. 5). A significant reduction in GPIC infectious load due to I-C treatment was accompanied by the reduction in severity of GPIC-induced local inflammation. Pathology scores were lower $(P<0.05$ on days $5,8,11$, and 13 post-infection) in the I-C pre-treated group in comparison with the animals receiving placebo treatment

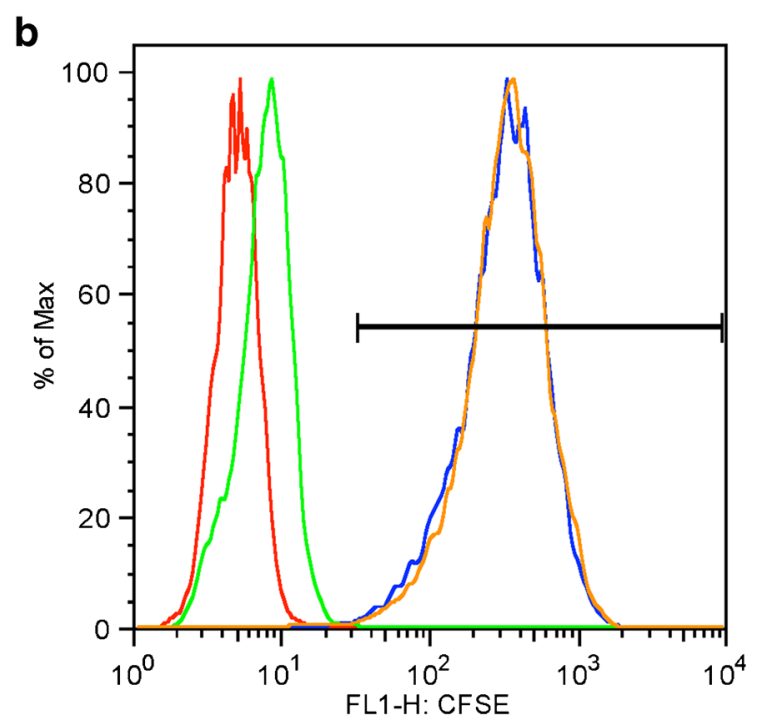

line) and non-infected $\mathrm{HCjE}$ incubated either with $\mathrm{I}-\mathrm{C}$ or with placebo solution (green line) were controls. All control cells were incubated under the same conditions ( $1 \mathrm{~h}$ at $37{ }^{\circ} \mathrm{C} / 5 \% \mathrm{CO}_{2}$ and $95 \%$ humidity) and treated in the same manner as $\mathrm{HCjE}$ cells infected with $\mathrm{CtB}$ in the presence of I-C or placebo (orange line). The gate containing CFSE+ cells is indicated on the histograms (black line)

(Fig. 5a). The I-C treatment did not shorten the period needed for complete clearance of the infection. Analysis of changes in chlamydial load during the post-infection period revealed significantly lower absolute numbers of GPIC EBs in I-C-treated guinea pigs on day $4(P<0.001)$, day $7(P<0.001)$, and day 14 $(P<0.005)$ post-infection in comparison with the corresponding placebo-treated animals (Fig. 5b).

\section{Discussion}

The results of the presented study show that I-C effectively reduces $\mathrm{CtB}$ infectivity by blocking chlamydial attachment to the epithelial host cells and might be a promising and affordable therapeutic agent against ocular chlamydial infections, especially for endemic trachoma areas.

The effects of a specific I-C treatment on the ocular chlamydial infection were assessed in vitro in $\mathrm{HCjE}$ cells and in vivo by using a model of inclusion conjunctivitis in guinea pigs. HCjE cells are considered a valuable and relevant model system for various kinds of ocular surface-targeted research. These cells are derived from healthy human conjunctiva and immortalized by abrogation of $\mathrm{p} 16$ control and $\mathrm{p} 53$ function before immortalization by expression of human telomerase reverse transcriptase (Gipson et al. 2003). Compared to cervical ME180 cells, which were originally isolated from a highly invasive cervical squamous cell carcinoma, $\mathrm{HCjE}$ cells are a more convenient system for testing due to their functional characteristics including similar mucin gene expression as native conjunctival cells (Gipson et al. 2003). An additional 

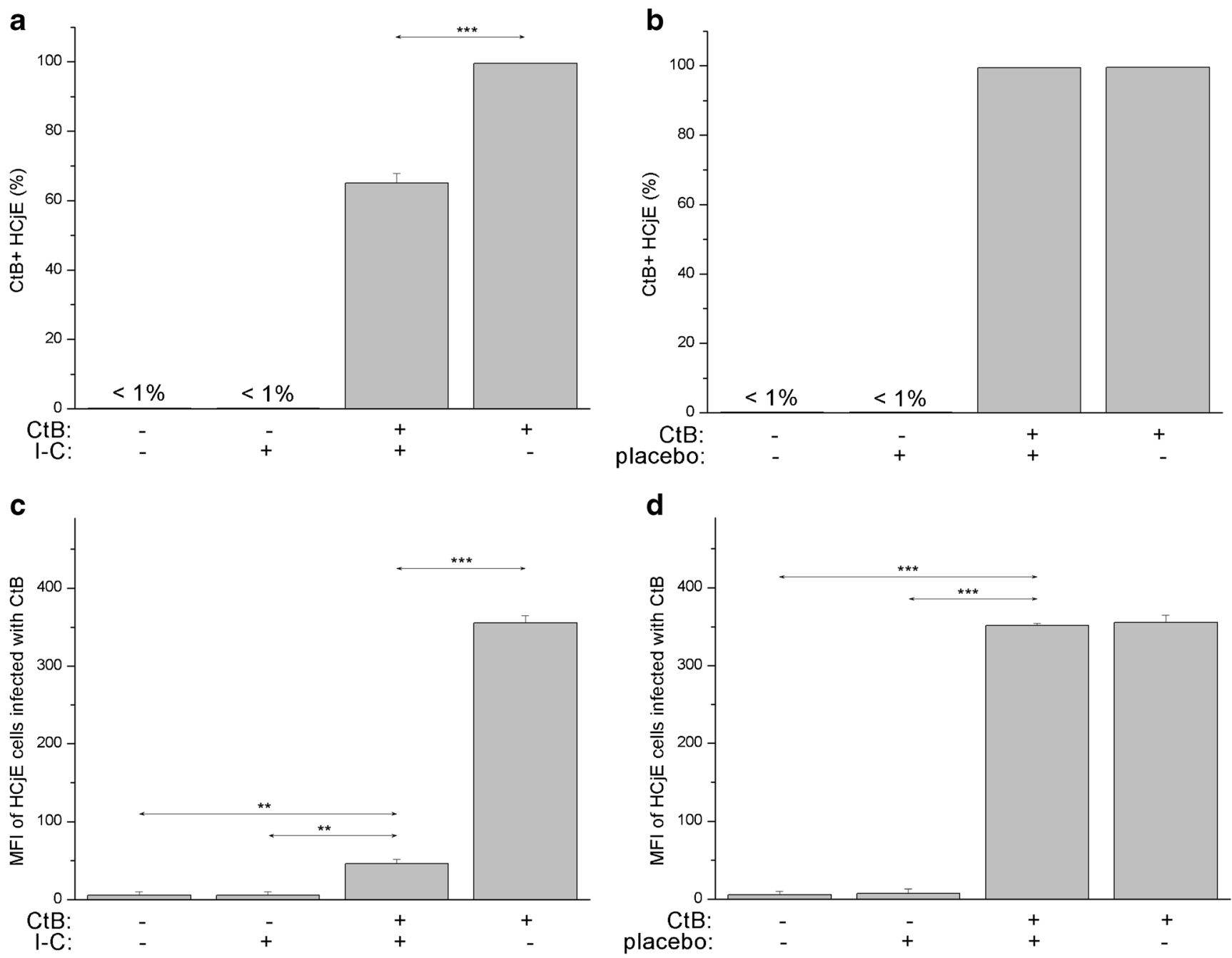

Fig. 2 Percentage of $\mathrm{CtB}+\mathrm{HCjE}$ cells within the total population of $\mathrm{HCjE}$ infected in vitro with CFSE-labeled $\mathrm{CtB}$ in the presence of I-C (a) and placebo (b) solution and the MFI of CFSE signal of HCjE cells exposed to treatments indicated below the plots $(\mathbf{c}, \mathbf{d})$. The gating of CFSE+ HCjE cells is indicated in Fig. 1 Three independent samples per

treatment were analyzed and results are presented as a mean value $\pm \mathrm{SE}$. Statistical significance between specific treatments is determined by oneway ANOVA followed by Bonferroni test $(* p<0.05$, ** $p<0.005$, *** $p<0.001)$. Compared groups are indicated by arrows

limitation of the study on ME180 cells was that they were reported to contain HPV DNA with greater homology to HPV-68 than HPV-18, which might speak against using these cells in infection experiments. Furthermore, the guinea pig model of inclusion conjunctivitis caused by GPIC we used in this study is an important animal model in the field of ocular chlamydial infection as the structural and functional organization of lymphoid tissue underlining the conjunctiva of guinea pigs highly resembles the one of the human ocular region (Rank 2007). This is the main advantage of a guinea pig inclusion conjunctivitis model over the other models of ocular infection of rodents.

Our results show that $\mathrm{I}-\mathrm{C}$ is able to reduce $C$. trachomatis infection in $\mathrm{HCjE}$ cells, which is in line with the effect of I-C seen for C. trachomatis serovar E in genital ME180 cells (Zaretzky et al. 1995). The reduced infectivity of the $C$.

trachomatis EBs in I-C-treated cells might be explained by the obstruction of their attachment to the I-C-treated host cells. It has been proposed that the inhibitory effect of SPs against $C$. trachomatis infection is non-specific and mediated by the strong negative charge of the SPs resulting in prevention of adherence of $C$. trachomatis to the host cell by charge repulsion (Zaretzky et al. 1995). The negative influence of I-C on EB attachment to the host cell could further explain the lower number of chlamydial IFUs and/or the smaller size of inclusions upon I-C treatment of host cells we found in our study. Our finding that inclusions in I-C-treated cells are smaller compared to untreated $\mathrm{CtB}$-infected cells suggests that there might be additional mechanisms involved that contribute to the antimicrobial effect of I-C.

Bacterial attachment to mucosal epithelial surfaces is the first step in the establishment of an infection and the 
a

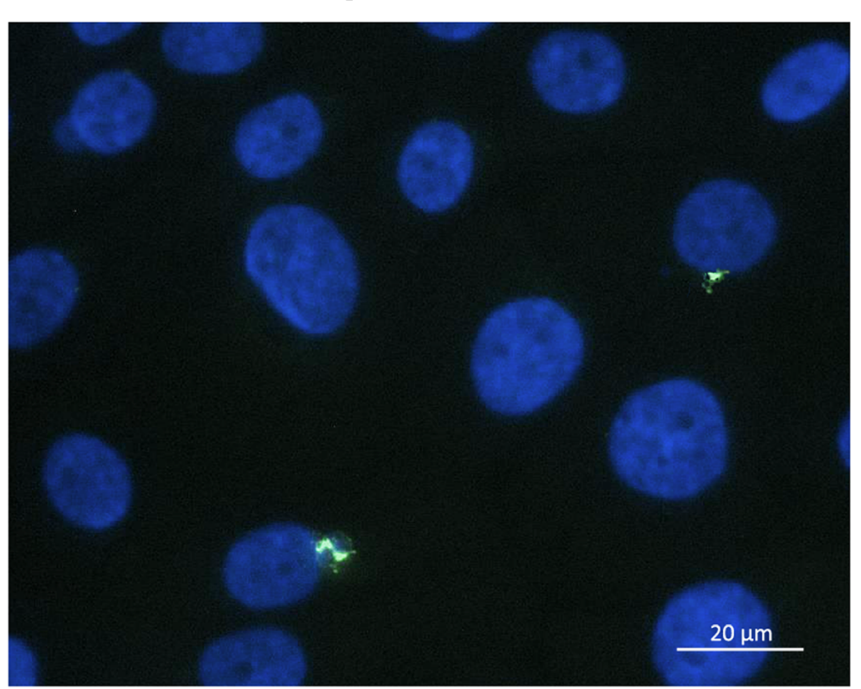

b

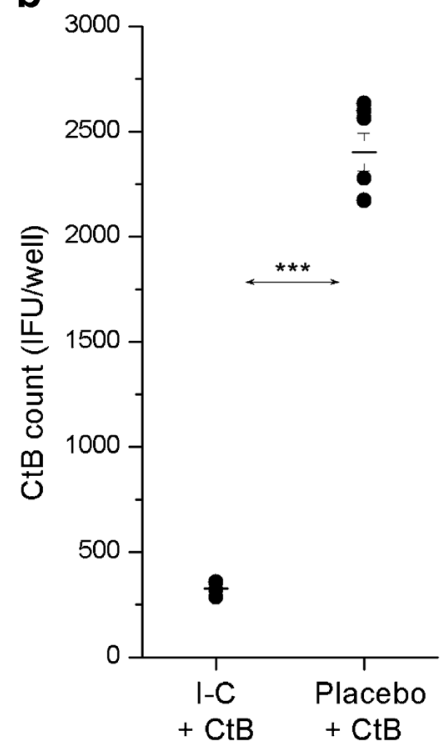

Fig. 3 Microscopic analysis of $\mathrm{HCjE}$ cells infected with $\mathrm{CtB}$ in the presence of I-C and placebo solution (a). $\mathrm{HCjE}$ cells were seeded at a density of 150.000 cells/well, infected with $1 \times 10^{4} \mathrm{IFU}$ of CtB, incubated in appropriate medium supplemented with I-C or placebo for $48 \mathrm{~h}$ and then collected for analyses. Representative images are shown. The

specificity of the pathogen-host cell interaction is determined by bacterial surface proteins (adhesins) and their receptors on the host cell surface. GAG structures on the mammalian cell surface play an important role in interactions with many microbial pathogens and are recognized also by Chlamydia (Zhang and Stephens 1992; Chen and Stephens 1994; Moelleken and Hegemann 2008). It has also been demonstrated that a chlamydial adhesin, the outer membrane protein B $(\mathrm{OmcB})$, from $C$. trachomatis as well as from GPIC, interacts with GAGs on the epithelial host cell surface (Stephens et al. 2001; Fadel and Eley 2007; Moelleken and Hegemann 2008). Interactions of carrageenans with host cell GAGs have been investigated and showed reduced sulfatase activity and

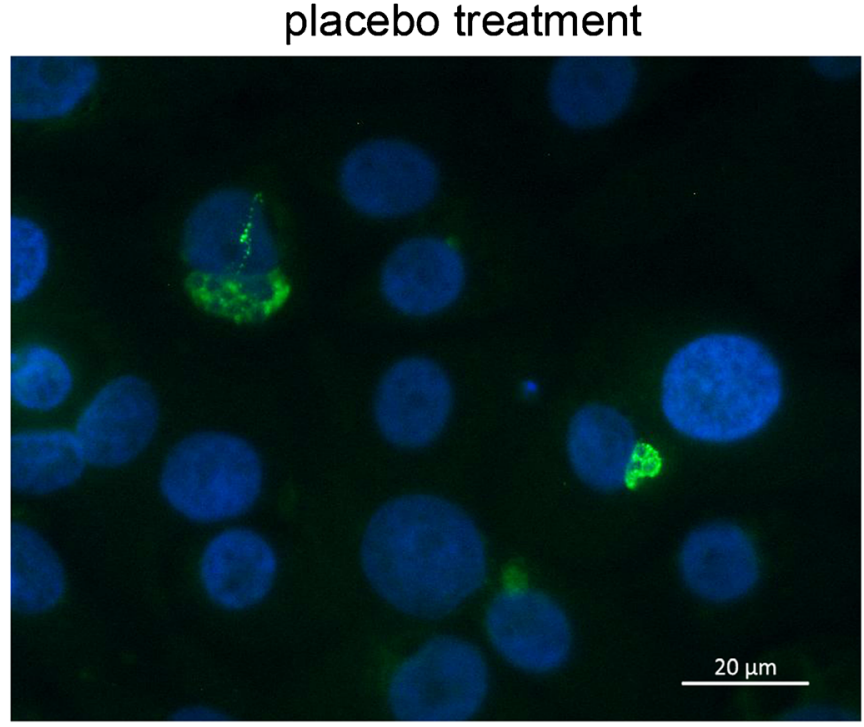

C

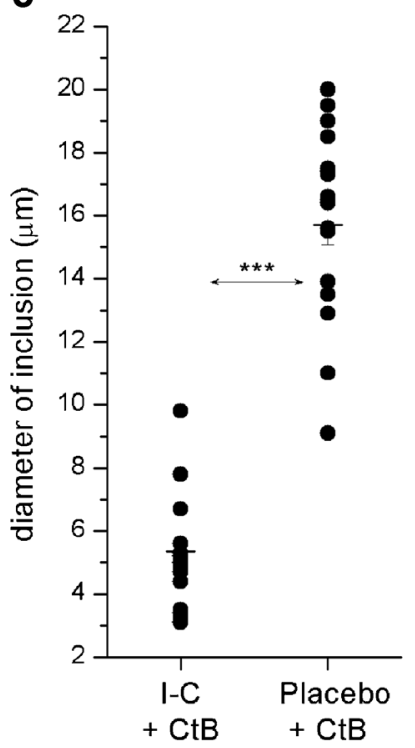

number (b) and the size (c) of $\mathrm{CtB}$ inclusions are determined. $\mathrm{CtB}$ inclusions are identified using FITC-labeled anti-Chlamydia LPS antibody (green dots). HCjE cells were counterstained with DAPI (blue). The significance of the recorded differences is determined by $t$ test for independent groups $(* * * P<0.001)$

redistribution of the cellular GAGs on the host cell surface with potential consequences for cell structure and function (Yang et al. 2012). Considering these findings, it can be hypothesized that I-C in our experiment competitively inhibits the interaction with GAGs on the epithelial cell surface or modulate their availability, which leads to reduced attachment and subsequent infection of the host cells.

Antiviral effects of I-C were investigated in animal models (Fernandez-Romero et al. 2012). It has been shown that I-C could effectively block HPV infection of HeLa and HaCat cells by inhibiting the interaction of the viral capsid with heparin sulfate proteoglycans at the host cell surface as well as that I-C and kappa-carrageenan are more 

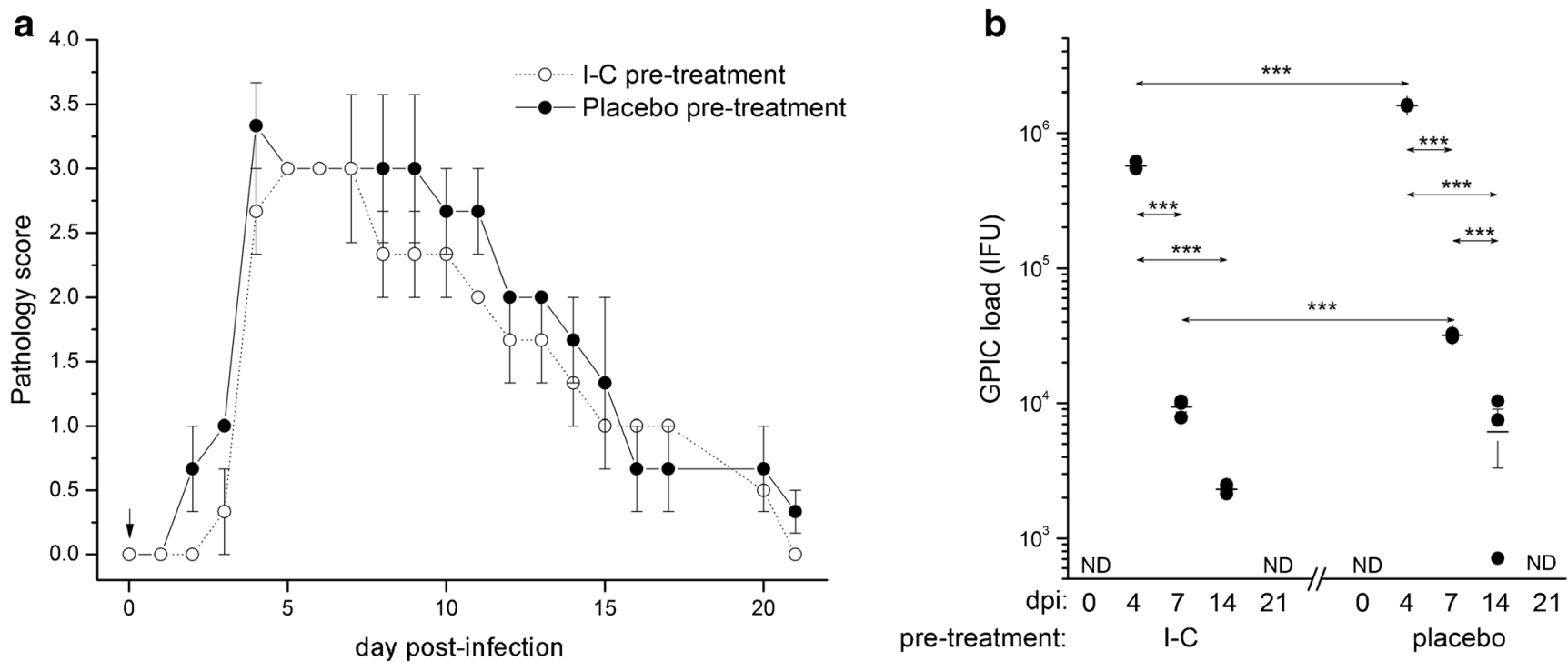

Fig. 4 Ocular pathology scores (a) and C. caviae load (b) in guinea pigs infected with a single ocular instillation of C. caviae $\left(1 \times 10^{4} \mathrm{IFU}\right) 2 \mathrm{~h}$ after I-C or placebo treatment. The start of infection is considered as day 0 and the timing of I-C or placebo pre-treatment is indicated by an arrow. The intensity of infection-induced inflammation is scored daily, while

C. caviae load is determined at previously selected control time-points (days $0,4,7,14$, and 21 post-infection). Statistical significance of the observed differences was evaluated using the two-way ANOVA test followed by Bonferroni test (compared groups indicated by arrows; $* P<0.05, * * P<0.005, * * * P<0.001)$

efficient in blocking HPV infection than other types of carrageenan (Buck et al. 2006). Differential efficiency of carrageenan to block HPV infection has also been reported showing a protective effect against HPV18 and HPV31 infections, but not against HPV16 and HPV45 (Cruz and Meyers 2013). The efficacy of carrageenan inhibition of dengue virus infection varied depending on the serotype tested (Talarico and Damonte 2016). Strain-specific efficacy of carrageenans depends on their properties but also on the characteristics of the infectious agent. It has been shown that the degree of sulfation has a major impact on the antiviral activity of SPs and that a specific positioning of sulfates might be important for the antiviral activity (Ghosh et al. 2009).
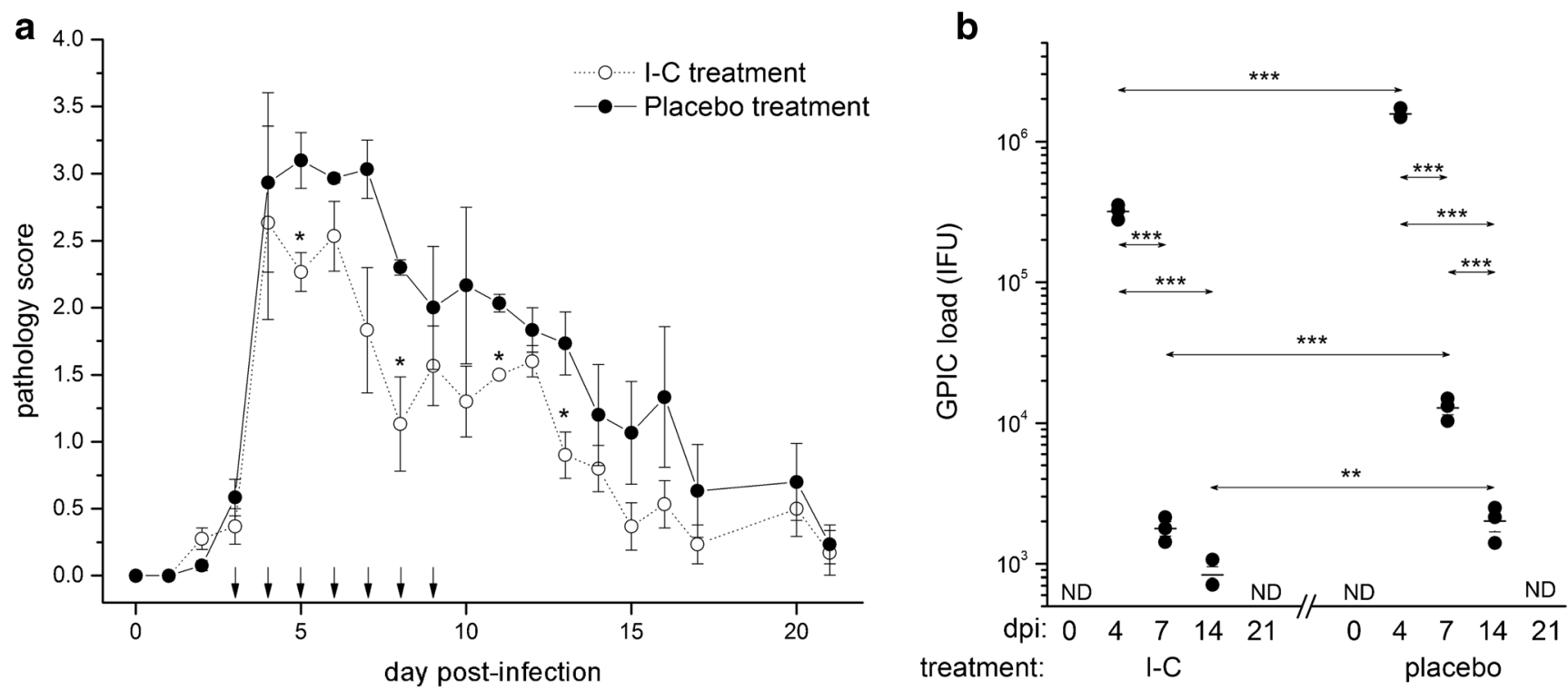

Fig. 5 Ocular pathology scores (a) and C. caviae load (b) in guinea pigs infected with a single ocular instillation of $C$. caviae $\left(1 \times 10^{4} \mathrm{IFU}\right)$ as well as guinea pigs treated during the acute phase of infection with I-C or placebo solution. The start of infection is considered as day 0 and the timing of I-C or placebo treatments (from day 3 to day 9 post-infection) are indicated with arrows. The intensity of infection-induced

inflammation is scored daily, while $C$. caviae load is determined at previously selected control time-points (days $0,4,7,14$, and 21 post-infection). Statistical significance of the observed differences was evaluated using the two-way ANOVA test followed by Bonferroni test (compared groups indicated by arrows; $* P<0.05, * * P<0.005$, and $* * * P<0.001)$ 
The in vitro obtained data on SPs as anti-chlamydial substances are in line with the strain-specific antiviral activity of SPs. It is demonstrated that the efficacy of SPs, including carrageenans, in inhibition of in vitro chlamydial infection depends on the Chlamydia serovar used (Zaretzky et al. 1995; Taraktchoglou et al. 2001).

Despite the existence of data implying a potential beneficial impact of SPs on the course of chlamydial infection, there is no clear evidence of a protective effect of SPs against chlamydial infection in vivo. Burillo et al. analyzed the protective capacity of SPs against chlamydial infections in a genital mouse model and did not find any protective effect (Burillo et al. 1998). Our results imply that topical I-C treatment could exert beneficial effects against ocular chlamydial infection but variation in dosage or application schedule must be carefully considered. Our results also imply that prolonged I-C application is required for a beneficial effect. Single I-C application $2 \mathrm{~h}$ before chlamydial infection has not resulted in a significant reduction in pathology intensity in comparison with placebo-treated animals. On the other hand, its daily application resulted in a significant lessening of pathology severity even though the treatment was started only at the acute phase of infection (day 3 post-infection) and not immediately after animals were infected with Chlamydia. This observation, together with our results of significantly less shedding of Chlamydia in I-C-treated animals compared with placebo-treated groups, additionally support the hypothesis that the anti-chlamydial activity of I-C might be attributed to the prevention of the initial $C$. trachomatis contact with the epithelial host cells. Furthermore, this finding could also implicate that I-C per se might exert a positive local immunomodulatory effect, which might consecutively contribute toward the inflammation lessening and the infection resolution.

In conclusion, our findings show that the application of $\mathrm{I}-\mathrm{C}$ reduces $\mathrm{CtB}$ infectivity in vitro and shedding of chlamydial EBs (GPIC) in vivo. Prolonged application of I-C might be needed for a significant improvement of the clinical picture. The reduction of shedding of infectious EBs is also of utter importance as it could contribute to a less effective transmission of chlamydial infection. Further studies, with the emphasis on the impact of I-C application schedule on the kinetics of a chlamydial infection are required to evaluate the full potential of I-C as a prophylactic and therapeutic treatment of ocular chlamydial infection.

Acknowledgments Open access funding provided by Medical University of Vienna. I-C for this study was kindly provided by Marinomed Biotechnologie GmbH, Vienna.

Funding This research was funded by the "Laura Bassi Centres of Expertise" program of the Austrian Federal Ministry of Economy through the Austrian Research Promotion Agency (FFG project number 822768); the Ministry of Education, Science, and Technological Development of the Republic of Serbia (grant number 172049).

Open Access This article is distributed under the terms of the Creative Commons Attribution 4.0 International License (http:// creativecommons.org/licenses/by/4.0/), which permits unrestricted use, distribution, and reproduction in any medium, provided you give appropriate credit to the original author(s) and the source, provide a link to the Creative Commons license, and indicate if changes were made.

\section{References}

Belij-Rammerstorfer S, Inic-Kanada A, Stojanovic M, Marinkovic E, Lukic I, Stein E, Montanaro J, Bintner N, Schurer N, Ghasemian E, Kundi M, Barisani-Asenbauer T (2016) Infectious dose and repeated infections are key factors influencing immune response characteristics in guinea pig ocular chlamydial infection. Microbes Infect $18: 254-262$

Bergsson G, Arnfinnsson J, Karlsson SM, Steingrimsson O, Thormar H (1998) In vitro inactivation of Chlamydia trachomatis by fatty acids and monoglycerides. Antimicrob Agents Chemother 42:2290-2294

Brown MA, Potroz MG, Teh SW, Cho NJ (2016) Natural products for the treatment of Chlamydiaceae infections. Microorganisms 4(4):39

Buck CB, Thompson CD, Roberts JN, Muller M, Lowy DR, Schiller JT (2006) Carrageenan is a potent inhibitor of papillomavirus infection. PLoS Pathog 2(7):e69

Burillo CA, Fontenot JD, Phillips DM (1998) Sulfated polysaccharides block chlamydia infection in vitro, but do not protect mice from vaginal inoculation. Microb Pathog 24(4):253-256

Caldwell HD, Kromhout J, Schachter J (1981) Purification and partial characterization of the major outer membrane protein of Chlamydia trachomatis. Infect Immun 31:1161-1176

Carlucci MJ, Ciancia M, Matulewicz MC, Cerezo AS, Damonte EB (1999) Antiherpetic activity and mode of action of natural carrageenans of diverse structural types. Antivir Res 43:93-102

Chen JC, Stephens RS (1994) Trachoma and LGV biovars of Chlamydia trachomatis share the same glycosaminoglycan-dependent mechanism for infection of eukaryotic cells. Mol Microbiol 11:501-507

Coggins C, Blanchard K, Alvarez F, Brache V, Weisberg E, Kilmarx PH, Lacarra M, Massai R, Mishell D Jr, Salvatierra A, Witwatwongwana P, Elias C, Ellertson C (2000) Preliminary safety and acceptability of a carrageenan gel for possible use as a vaginal microbicide. Sex Transm Infect 76:480-483

Cruz L, Meyers C (2013) Differential dependence on host cell glycosaminoglycans for infection of epithelial cells by high-risk HPV types. PLoS One 8(7):e68379

Daglia M (2012) Polyphenols as antimicrobial agents. Curr Opin Biotechnol 23:174-181

Damonte EB, Matulewicz MC, Cerezo AS (2004) Sulfated seaweed polysaccharides as antiviral agents. Curr Med Chem 11:2399-2419

Eccles R, Winther B, Johnston SL, Robinson P, Trampisch M, Koelsch S (2015) Efficacy and safety of iota-carrageenan nasal spray versus placebo in early treatment of the common cold in adults: the ICICC trial. Respir Res 16:121

ECDC (2015) STI trends in Europe: chlamydia rates stabilise while gonorrhoea numbers go up - See more at: http://ecdc.europa.eu/en/ press/news/ layouts/forms/News_DispForm.aspx?ID=1285\&List= 8db7286c-fe 2d-476c-9133-18ff4cb1b568\#sthash.UTipMXVx. dpuf. http://ecdc.europa.eu/en/press/news/_layouts/forms/News DispForm.aspx $?$ ID $=1285 \&$ List $=8 \mathrm{db} 7286 \mathrm{c}-\mathrm{fe} 2 \mathrm{~d}-476 \mathrm{c}-9133-$ 18ff4cb1b568. Accessed 05.02.2017 2016 
Fadel S, Eley A (2007) Chlamydia trachomatis OmcB protein is a surface-exposed glycosaminoglycan-dependent adhesin. J Med Microbiol 56:15-22

FDA SCOGS (Select Committee on GRAS Substances) (n.d.) http:// www.accessdata.fda.gov/scripts/fdcc/?set=SCOGS

Fernandez-Romero JA, Abraham CJ, Rodriguez A, Kizima L, JeanPierre N, Menon R, Begay O, Seidor S, Ford BE, Gil PI, Peters J, Katz D, Robbiani M, Zydowsky TM (2012) Zinc acetate/ carrageenan gels exhibit potent activity in vivo against high-dose herpes simplex virus 2 vaginal and rectal challenge. Antimicrob Agents Chemother 56:358-368

Filipovic A, Ghasemian E, Inic-Kanada A, Lukic I, Stein E, Marinkovic E, Djokic R, Kosanovic D, Schuerer N, Chalabi H, BelijRammerstorfer S, Stojanovic M, Barisani-Asenbauer T (2017) The effect of infectious dose on humoral and cellular immune responses in Chlamydophila caviae primary ocular infection. PLoS One 12(7): e0180551

Frick KD, Lietman TM, Holm SO, Jha HC, Chaudhary JS, Bhatta RC (2001) Cost-effectiveness of trachoma control measures: comparing targeted household treatment and mass treatment of children. Bull World Health Organ 79:201-207

Ghosh T, Pujol CA, Damonte EB, Sinha S, Ray B (2009) Sulfated xylomannans from the red seaweed Sebdenia polydactyla: structural features, chemical modification and antiviral activity. Antivir Chem Chemother 19:235-242

Gipson IK, Spurr-Michaud S, Argueso P, Tisdale A, Ng TF, Russo CL (2003) Mucin gene expression in immortalized human corneallimbal and conjunctival epithelial cell lines. Invest Ophthalmol Vis Sci 44:2496-2506

Gonzalez ME, Alarcon B, Carrasco L (1987) Polysaccharides as antiviral agents: antiviral activity of carrageenan. Antimicrob Agents Chemother 31:1388-1393

Grassauer A, Weinmuellner R, Meier C, Pretsch A, Prieschl-Grassauer E, Unger H (2008) Iota-carrageenan is a potent inhibitor of rhinovirus infection. Virol J 5:107

Klimyte EM, Smith SE, Oreste P, Lembo D, Dutch RE (2016) Inhibition of human metapneumovirus binding to heparan sulfate blocks infection in human lung cells and airway tissues. J Virol 90:9237-9250

Lahaye M (2001) Developments on gelling algal galactans, their structure and physico-chemistry. J Appl Phycol 13:173-184

Leibbrandt A, Meier C, Konig-Schuster M, Weinmullner R, Kalthoff D, Pflugfelder B, Graf P, Frank-Gehrke B, Beer M, Fazekas T, Unger $\mathrm{H}$, Prieschl-Grassauer E, Grassauer A (2010) Iota-carrageenan is a potent inhibitor of influenza A virus infection. PLoS One 5(12): e14320

Levendosky K, Mizenina O, Martinelli E, Jean-Pierre N, Kizima L, Rodriguez A, Kleinbeck K, Bonnaire T, Robbiani M, Zydowsky TM, O'Keefe BR, Fernandez-Romero JA (2015) Griffithsin and carrageenan combination to target herpes simplex virus 2 and human papillomavirus. Antimicrob Agents Chemother 59:7290-7298

Lietman T, Dawson C, Osaki S (1998) Ocular chlamydial infections. Int Ophthalmol Clin 38:125-135

Luo Z, Tian D, Zhou M, Xiao W, Zhang Y, Li M, Sui B, Wang W, Guan $\mathrm{H}$, Chen H, Fu ZF, Zhao L (2015) Lambda-carrageenan P32 is a potent inhibitor of rabies virus infection. PLoS One 10(10): e0140586

Mariotti SP, Pascolini D, Rose-Nussbaumer J (2009) Trachoma: global magnitude of a preventable cause of blindness. Br J Opthamol 93: $563-568$

McKim JM Jr, Baas H, Rice GP, Willoughby JA Sr, Weiner ML, Blakemore W (2016) Effects of carrageenan on cell permeability, cytotoxicity, and cytokine gene expression in human intestinal and hepatic cell lines. Food Chem Toxicol 96:1-10

Moelleken K, Hegemann JH (2008) The chlamydia outer membrane protein $\mathrm{OmcB}$ is required for adhesion and exhibits biovar-specific differences in glycosaminoglycan binding. Mol Microbiol 67:403-419
Molleken K, Schmidt E, Hegemann JH (2010) Members of the Pmp protein family of Chlamydia pneumoniae mediate adhesion to human cells via short repetitive peptide motifs. Mol Microbiol 78: 1004-1017

Necas J, Bartosikova L (2013) Carrageenan: a review. Vet Mad 58(4): $187-205$

Norman J (2002) Epidemiology of female genital Chlamydia trachomatis infections. Best Pract Res Clin Obstet Gynaecol 16:775-787

Rahn C, Marti H, Frohns A, Frohns F, Blenn C, Leonard CA, BarisaniAsenbauer T, Stein E, Borel N (2016) Water-filtered infrared A reduces chlamydial infectivity in vitro without causing ex vivo eye damage in pig and mouse models. J Photochem Photobiol B 165: $340-350$

Rank R (2007) Chlamydial diseases. In: Fox JG (ed) The mouse in biomedical research diseases. Elsevier, Amsterdam, pp 327-348

Rank RG, Dascher C, Bowlin AK, Bavoil PM (1995) Systemic immunization with Hsp60 alters the development of chlamydial ocular disease. Invest Ophthalmol Vis Sci 36:1344-1351

Rank RG, Whittum-Hudson JA (1994) Animal models for ocular infections. Methods Enzymol 235:69-83

Roberts JN, Buck CB, Thompson CD, Kines R, Bernardo M, Choyke PL, Lowy DR, Schiller JT (2007) Genital transmission of HPV in a mouse model is potentiated by nonoxynol- 9 and inhibited by carrageenan. Nat Med 13:857-861

Schnitger K, Njau F, Wittkop U, Liese A, Kuipers JG, Thiel A, Morgan MA, Zeidler H, Wagner AD (2007) Staining of Chlamydia trachomatis elementary bodies: a suitable method for identifying infected human monocytes by flow cytometry. J Microbiol Methods 69:116-121

Stein E, Inic-Kanada A, Belij S, Montanaro J, Bintner N, Schlacher S, Mayr UB, Lubitz W, Stojanovic M, Najdenski H, BarisaniAsenbauer T (2013) In vitro and in vivo uptake study of Escherichia coli Nissle 1917 bacterial ghosts: cell-based delivery system to target ocular surface diseases. Invest Ophthalmol Vis Sci 54:6326-6333

Stephens RS, Koshiyama K, Lewis E, Kubo A (2001) Heparin-binding outer membrane protein of chlamydiae. Mol Microbiol 40:691-699

Talarico LB, Damonte EB (2016) Characterization of in vitro dengue virus resistance to carrageenan. J Med Virol 88:1120-1129

Talwar GP, Garg S, Dhar V, Chabra R, Ganju A, Upadhyay SN (1995) Praneem polyherbal cream and pessaries with dual properties of contraception and alleviation of genital infections. Curr Sci 68:437-440

Taraktchoglou M, Pacey AA, Turnbull JE, Eley A (2001) Infectivity of Chlamydia trachomatis serovar LGV but not $\mathrm{E}$ is dependent on host cell heparan sulfate. Infect Immun 69:968-976

Taylor HR, Burton MJ, Haddad D, West S, Wright H (2014) Trachoma. Lancet 384:2142-2152

Vera J, Castro J, Gonzalez A, Moenne A (2011) Seaweed polysaccharides and derived oligosaccharides stimulate defense responses and protection against pathogens in plants. Mar Drugs 9:2514-2525

WHO (2014) Alliance for the global elimination of blinding trachoma by the year 2020. Wkly Epidemiol Rec 89:421-428

Yamashita S (2001) In vitro bacteriostatic effects of dietary polysaccharides. Food Sci Technol Res 7:262-264

Yang B, Bhattacharyya S, Linhardt R, Tobacman J (2012) Exposure to common food additive carrageenan leads to reduced sulfatase activity and increase in sulfated glycosaminoglycans in human epithelial cells. Biochimie 94:1309-1316

Yasin B, Harwig SS, Lehrer RI, Wagar EA (1996) Susceptibility of Chlamydia trachomatis to protegrins and defensins. Infect Immun 64:709-713

Zaretzky FR, Pearce-Pratt R, Phillips DM (1995) Sulfated polyanions block Chlamydia trachomatis infection of cervix-derived human epithelia. Infect Immun 63:3520-3526

Zhang JP, Stephens RS (1992) Mechanism of C. trachomatis attachment to eukaryotic host cells. Cell 69:861-869 